\title{
Photothermal Treatment of Human Pancreatic Cancer Using PEGylated Multi-Walled Carbon Nanotubes Induces Apoptosis by Triggering Mitochondrial Membrane Depolarization Mechanism
}

\author{
Teodora Mocan ${ }^{1 *}$, Cristian T. Matea $^{1^{*}}$, Iulia Cojocaru ${ }^{1 *}$, Ioana Ilie ${ }^{1^{*}}$, Flaviu A. Tabaran ${ }^{2 *}$, Florin Zaharie ${ }^{1}$,
} Cornel Iancu ${ }^{1}$, Dana Bartos ${ }^{1}$, Lucian Mocan ${ }^{1 *} \bowtie$

1. Gastroenterology Institute; Department of Nanomedicine, “Iuliu Hatieganu” University of Medicine and Pharmacy, 19-21 Croitorilor Street, Cluj-Napoca, Romania;

2. Department of Pathology, University of Agricultural Sciences and Veterinary Medicine, Faculty of Veterinary Medicine, 3-5 Manastur Street, 400372 Cluj-Napoca, Romania.

*These authors contributed equally to this work.

$\square$ Corresponding author: Mocan Lucian Tel: +40264-439696 Fax: +40264-439696 Email address: lucian.mocan@umfcluj.ro.

() Ivyspring International Publisher. This is an open-access article distributed under the terms of the Creative Commons License (http://creativecommons.org/ licenses/by-nc-nd/3.0/). Reproduction is permitted for personal, noncommercial use, provided that the article is in whole, unmodified, and properly cited.

Received: 2014.04.23; Accepted: 2014.07.23; Published: 20I4.09.05

\begin{abstract}
Pancreatic cancer (PC) is one of the most lethal solid tumor in humans, with an overall 5-year survival rate of less than $5 \%$. Thermally active carbon nanotubes have already brought to light promising results in PC research and treatment.

We report here the construct of a nano-biosystem based on multi-walled carbon nanotubes and polyethylene glycol (PEG) molecules validated through AFM, UV-Vis and DLS. We next studied the photothermal effect of these PEG-ylated multi-walled carbon nanotubes $(5,10$ and $50 \mu \mathrm{g} / \mathrm{mL}$, respectively) on pancreatic cancer cells (PANC-I) and further analyzed the molecular and cellular events involved in cell death occurrence. Using cell proliferation, apoptosis, membrane polarization and oxidative stress assays for ELISA, fluorescence microscopy and flow cytometry we show here that hyperthermia following MWCNTs-PEG laser mediated treatment $(808 \mathrm{~nm}, 2 \mathrm{~W})$ leads to mitochondrial membrane depolarization that activates the flux of free radicals within the cell and the oxidative state mediate cellular damage in PC cells via apoptotic pathway. Our results are of decisive importance especially in regard with the development of novel nano-biosystems capable to target mitochondria and to synergically act both as cytotoxic drug as well as thermally active agents in order to overcome one of the most common problem met in oncology, that of intrinsic resistance to chemotherapeutics.
\end{abstract}

Key words: carbon nanotubes, pancreatic cancer, PEG functionalization, photothermal ablation, apoptosis, mitochondrial therapy.

\section{Background}

Pancreatic cancer (PC) is one of the most lethal solid tumors in humans, with an overall 5-year survival rate of less than 5\% (1). Surgical excision of the primary tumor in these patients provides clinical benefits such as prolongation of survival. Because at the time of the first symptoms, most PC patients have advanced-stage disease, surgical resection and treatment with curative intention is possible in only $20 \%$ of 
the cases. PC is also one of the most highly chemoresistant cancer and the lack of specific and effective cytostatics contributes to its poor survival rates (2).

The development of drug delivery systems represents an exciting opportunity to improve PC outcome. A drug delivery carrier represents an active molecule attached to a delivery antibody with affinity for specific cell surface epitopes. The delivery vehicle has the ability to concentrate only inside the targeted cell. The use of nanoparticles as anti-cancer delivery carriers is a very promising approach because these nanoscale materials can fulfil all the requirements needed for an ideal oncologic agent. Development of specific and sensitive site-targeted thermally active bio-nanosystems may open new avenues in the field of cancer targeting by nanoparticles (3). In oncology, treatments based on thermal energy are superior to conventional techniques, since they are minimally invasive and clinically feasible, having great capacity to treat tumors in certain anatomical sites where surgical resection is not technically possible. Simple heating techniques such as ultrasound or radiofrequency ablation cannot differentiate between tumor and healthy tissue, often producing necrosis of a functional part of an organ. The development of nanomedicine research determined the rapid expansion of a variety of nanostructures with special optical properties and various biomedical applications $(4,5)$. Photothermal therapy employing carbon nanotubes thermally activated with near-infrared (NIR) laser can destroy cells at a molecular level and thus to produce selective necrosis of malignant cells (6). Following NIR irradiation, an elevated local heating in carbon nanotubes was observed attaining a threshold temperature with a nonlinear effect, producing irreversible destruction in biological systems. Therefore, the nanophotothermolysis is performed at a microscale level at lower intensities, rendering a minimally invasive therapy (7). Thermally active carbon nanotubes have already brought to light promising results in PC research and treatment $(8,9)$. Despite these promising results, some fundamental toxicities and biological interactions hamper CNT deployment to the clinic (5, 10, 11). These biological interactions include uptake by the reticuloendothelial system (RES), in which NPs are rapidly washed out of circulation, and the lack of selectivity of these compounds for a certain anatomical region (12). Moreover, because of aggregation phenomenon CNT may produce capillary occlusion in vital organs. Addition of hydrophilic molecules such as PEG (a coiled polymer of repeating ethylene ether units) onto the surface of carbon nanotubes (PEG ylation) can significantly enhance their solubility and help evading macrophage-mediated uptake thus blocking the enzymatic degradation of the carriers while in systemic circulation (13-15).

We report here the construct of a nano-bioystem based on multi-walled carbon nanotubes and polyethylene glycol (PEG) molecules to target pancreatic cancer cells for laser ablation purposes. We next studied the photothermal effect of these PEG-ylated multi-walled carbon nanotubes on PANC-1 PC cells and further analyzed the molecular events involved in cellular death occurrence. Here we show that LASER treatment following administration of PEG-ylated multi-walled carbon nanotubes exhibit increased mitochondrial membrane depolarization resulting in immediate cellular apoptosis. Activation of mitochondrial membrane permeabilization leading to mitochondrial programmed apoptosis by various pharmacological agents holds great hopes for future cancer therapies. Therefore our results could open new avenues in the field of mitochondrial therapy against human cancers.

\section{Materials and methods}

\section{Non-covalent functionalization of MWNTs with PEG}

A straight forward approach of carbon nanotube functionalization by means of simple ultrasonication was employed. Multiwall carbon nanotubes and polyethylene glycol $\mathrm{M}_{\mathrm{w}} 400$ were purchased from Sigma-Aldrich (Darmstadt, Germany). 5mg of pristine MWCNT were dispersed in 100 mL PEG solution (1\% $\mathrm{v} / \mathrm{v}$ ) with the aid of a Virtis 1100 sonicator (3x3min). Removal of unbound and agglomerated MWCNTs was performed by centrifugation at $7000 \mathrm{RPM} / 5 \mathrm{~min}$ where only the supernatant was retained. Removal of agglomerates of unbound MWCNT was than performed by centrifugation and selective dimension filtering. The final concentration of MWCNTs-PEG solution was approximately $50 \mu \mathrm{g} / \mathrm{mL}$, as determined by UV-VIS reading. For the experiment, seriate distilled water-diluted samples were prepared $(5,10$ $\mu \mathrm{g} / \mathrm{mL}$ ).

The samples prepared presented good stability and no agglomeration was observed for the samples stored at room temperature for several weeks. In order to characterize the obtained MWCNTs-PEG complexes UV-Vis spectroscopy, Dynamic Light Scattering and atomic force microscopy (AFM) were used. The UV-Vis spectra were recorded on a Shimadzu UV-1800 instrument. Dynamic Light Scattering (DLS) analysis was performed on a Zetasizer Nano S90 (Malvern Instruments, UK). Measurements were conducted at $25^{\circ} \mathrm{C}$ at a $90^{\circ}$ scattering angle in triplicate.

Atomic force microscopy measurements were performed on a Workshop TT-AFM ${ }^{\mathrm{TM}}$ in vibrating mode using super sharp ACTA-SS cantilevers (Ap- 
pnano Mountain View, CA, USA). AFM images were processed using Gwyddion ${ }^{\circledR}$ software.

\section{In vitro Augmented-Darkfield light microscopy imaging of nanoparticle interaction with PANCI cells}

To observe the cellular uptake of PEG-MWCNT and morphological changes suffered by tumoral cells after exposure to nanomaterials a CytoViva150 Ultrahigh Resolution Imaging (URI) System (Auburn, AL) attached to a Olympus BX-43 microscope was used. Images were collected with a DAGE-MTI XL16 digital camera (DAGE-MTI, Michigan City) as $1600 \times 1200$ pixel/ 8 bit and processed with Exponent 7 Software. The CytoViva enhanced dark-field based illumination system due to the high signal-to-noise allows the direct intracellular visualization of particles with dimensions less than the wavelength of light as MWCNT.

\section{Cell culture}

Human pancreatic adenocarcinoma cells (PANC-1) were grown using Dulbecco's modified Eagle's medium (DMEM) with 10\% fetal bovine serum (FBS) and 2mM Glutamine supplement). Standard conditions were assured for optimal growth $\left(5 \% \mathrm{CO}_{2}, 37^{\circ} \mathrm{C}\right)$. Sub-confluent cultures were split and seeded at appropriate density $\left(2-4 \times 10,000\right.$ cells $\left./ \mathrm{cm}^{2}\right)$ using $0.25 \%$ trypsin/EDTA, according to manufacturer's protocols.

\section{Exposure to MWCNTs-PEG}

When reaching the growth log-phase, test samples' cells were exposed to MWCNTs-PEG by medium removal and the addition of MWCNTs-PEG solution in appropriate concentrations $(5,10$ and 50 $\mu \mathrm{g} / \mathrm{mL}$, respectively). A volume of $300 \mu \mathrm{l} /$ well for 4 chamber slides (Labtek), $1 \mathrm{ml} /$ well for 6 well plates or $100 \mu \mathrm{l} /$ well for 96 well plates were used. Next, one hour incubation $\left(5 \% \mathrm{CO}_{2}, 37^{\circ} \mathrm{C}\right)$ was allowed for exposure, followed by irradiation in test samples. Control sample was exposed to appropriate culture medium and has not been subject to additional laser excitation.

\section{Laser treatment}

Each MWCNTs-PEG-exposed culture sample (5, 10 and $50 \mu \mathrm{g} / \mathrm{mL}$, respectively) was photoexcited for 3 minutes, using an $808 \mathrm{~nm}$ laser, $2 \mathrm{~W} / \mathrm{cm}^{2}$ power. Removal of exposure solution was performed before irradiation and vertical position was assured for the laser diode ( $2 \mathrm{~cm}$ above the adherent cell layer). To assure equivalence of laser excitation regardless the surface of the seeding well designed for various analysis techniques, triple irradiation positions, marked by equal divisions of the well's surface were used for samples seeded in 4-well chambers (further analysed by means of microscopically or by flow cytometry techniques) and single irradiation position was used for samples seeded in the 96 well chamber (for MTT evaluation).

\section{MTT assay}

Mitochondrial dehydrogenase activity was used to measure cell growth and viability by using cleavage of 3-[4, 5-dimethylthiazol-2-yl]-2, 5-diphenyl tetrazolium bromide (MTT). For performing the assay (Sigma-Aldrich), cells were seeded into a 96 well plate. Briefly, following exposure and irradiation, cells were incubated with MTT solution $\left(3 \mathrm{hrs}, 37^{\circ} \mathrm{C}\right)$. Next, formazan crystals were dissolved (gentle stirring, RT). Absorbance was read by spectrophotometry at $570 \mathrm{~nm}$ with substraction of background absorbance $(690 \mathrm{~nm})$ using the fluorescence modulus of a Perkin Elmer Viktor 2 multilabel multitask plate reader. Experiments were performed in triplicate. Results were expressed as percentage calculated from non-exposed group's absorbance.

\section{Annexin-V Cy3 Apoptosis Detection Assay}

Identification of apoptotic cells was performed by binding of Cy3-(fluorescently conjugated) Annexin $\mathrm{V}$ to phosphatidyl-serine (PS) groups on the outer surface of the cell membrane. Cells were seeded into 4-chamber glass slide (Milipore). Briefly, immediately after exposure and irradiation, cells were incubated with AnnexinV-Cy3 (5 min, dark), followed by microscopic examination using a rhodamine filter.

\section{Mitochondrial Membrane Potential Detection Assay}

Cells were seeded into 4-chamber glass slide.(Milipore) until reaching the desidered $80 \%$ confluence. According to producer's protocol, sample treatment was followed by washing and incubation with the special Dual Detection Reagent (15 min, RT, dark). Next, samples were carefully examined using microscopical techniques. Green-stained depolarized mitochondrias were detected using FITC filter set $(E x / E m=485 / 530 \mathrm{~nm})$ while the orange-stained energized mitochondria were identified using a rhodamine filter $(\mathrm{Ex} / \mathrm{Em}=540 / 570 \mathrm{~nm})$.

\section{Microscopic analyses}

All microscopical analyzes were performed using a high-performance FSX-100 Olympus Microscope. Phase-contrast and multi-excitation filter (R, B, $\mathrm{G})$ fluorescence detection modes were used.

\section{Total ROS Detection Assay}

Seeding the cells on 6-well plates (Cornig Costar, Fisher Scientific) one day prior to the experiment was 
performed to assure $50-70 \%$ confluence. Staining for flowcitometric analysis was performed using total ROS Detection Kit ( Enzo Life Sciences). Briefly, following treatment, samples were suctioned to remove media and detached using sterile scraping technique. Collection of cells in $5 \mathrm{~mL}$ polystyrene tubes and staining $\left(30 \mathrm{~min}, 37^{\circ} \mathrm{C}\right.$ ) with the ROS detection solution provided by the manufacturer was performed. Reactive oxygen species accumulation was evaluated using a FACSCalibur flow cytometer (Becton Dickinson, San Jose, CA, USA) with green (FL1 channel-530 $\mathrm{nm})$ The Total ROS Detection Kit (Enzo Life Sciences) was used for cell cultures staining following administration of MWCNTs-PEG and Laser irradiation. Next, suspensions of Panc- 1 cells were analyzed by flow cytometry in accordance with the manufacturer's instructions.

\section{Image analysis}

Quantification of Annexin V and membrane polarization levels were performed in Image $(\mathrm{NIH}$, ver. $1.43 \mathrm{u}$ ) by applying a linear stretch of the pixel intensity histogram corresponding to each slice in the $z$ stack to adjust the number of low and high intensity pixels thus increasing the contrast. To remove the spaces between each slice of the z-stack and to create a single image from all slices the image was further converted into a $\mathrm{z}$ projection. A $7 \times 7$ top-hat filter was further applied, followed by a median filter and a thresholding step. The thresholded image was then converted to a binary image, which results in white structures (Annexin V/green fluorescence) on a black background that was further analyzed in ImageJ.

\section{Results}

\section{Characterization of MWCNTs-PEG bionanosystem}

To elucidate the molecular mechanisms behind this nanomediated induced thermal necrosis we have functionalized multi-walled carbon nanotubes with polyethylene glycol (PEG) for PC cell laser treatment (Fig.1). This polyether compound is commonly used in nanomedicine applications for nanomaterials coating to its increased biocompatibility in biological systems. UV-Vis spectra (Fig.2a) were recorded using a Shimadzu UV-1800 instrument. All spectra registered were analysed and normalized using the Origin Lab 8.5 software. Bundled CNTs do not present $\mathrm{UV}-\mathrm{Vis}$ spectral fingerprints, most probably due to charge transfer between the respective nanotubes. However, in well dispersed CNT solutions an absorption peak for the nanotubes can be recorded between 200-300 nm (16). The UV-Vis spectra of the MWCNTs-PEG sample shown in figure 2a has a $\lambda_{\max }=265 \mathrm{~nm}$. In order to establish the hydrodynamic diameter of the pegylated MWCNTs we next employed the DLS technique (Fig.2b). Even though DLS analysis mainly provides information about sphere shaped particles, the technique can provide hydrodynamic diameter information in the case of carbon nanotubes $(17,18)$.

The aqueous solution containing MWCNTs-PEG presented its self as monodisperse and stable. The good stability of MWCNTs-PEG in water can be attributed to the oxygen-containing glycol chain, which is able to form hydrogen bonds with the water molecules (18).

Using DLS analysis we showed that particle size distribution of MWCNTs-PEG bundles in aqueous media ranged from 298-728 $\mathrm{nm}$. As seen in figure 2c following functionalization with PEG, AFM measurements of the PEGylated MWCNTs showed an increased diameter for these bio-nanosystems ranging from $44-46 \mathrm{~nm}$. This is due to the non-covalent binding of PEG onto the surface of the MWCNTs.
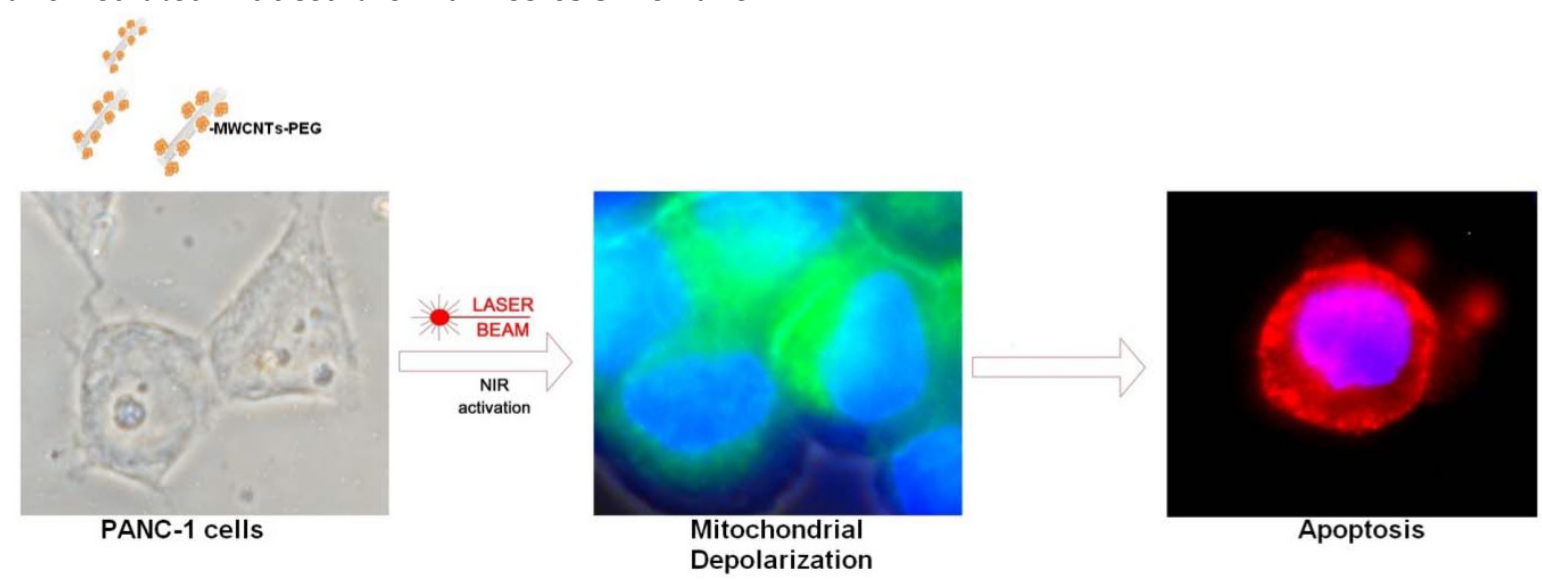

Figure I: Schematic illustration of cellular mechanisms involved in nanophotothermolysis of pancreatic cancer cells mediated by MWCNTs-PEG. Following cellular exposure to various concentrations of PEG-ylated MWCNTs PANC-I cells were irradiated using $808 \mathrm{~nm}, 2 \mathrm{~W}$ Laser beam. 
a

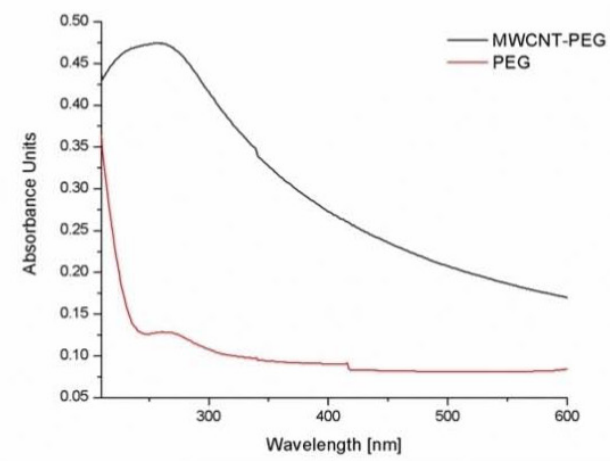

C

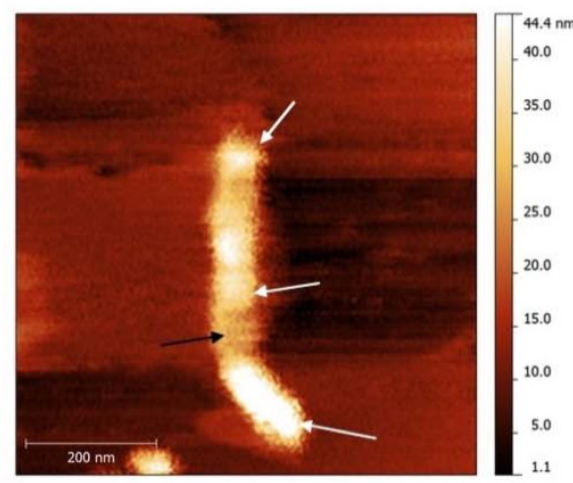

b
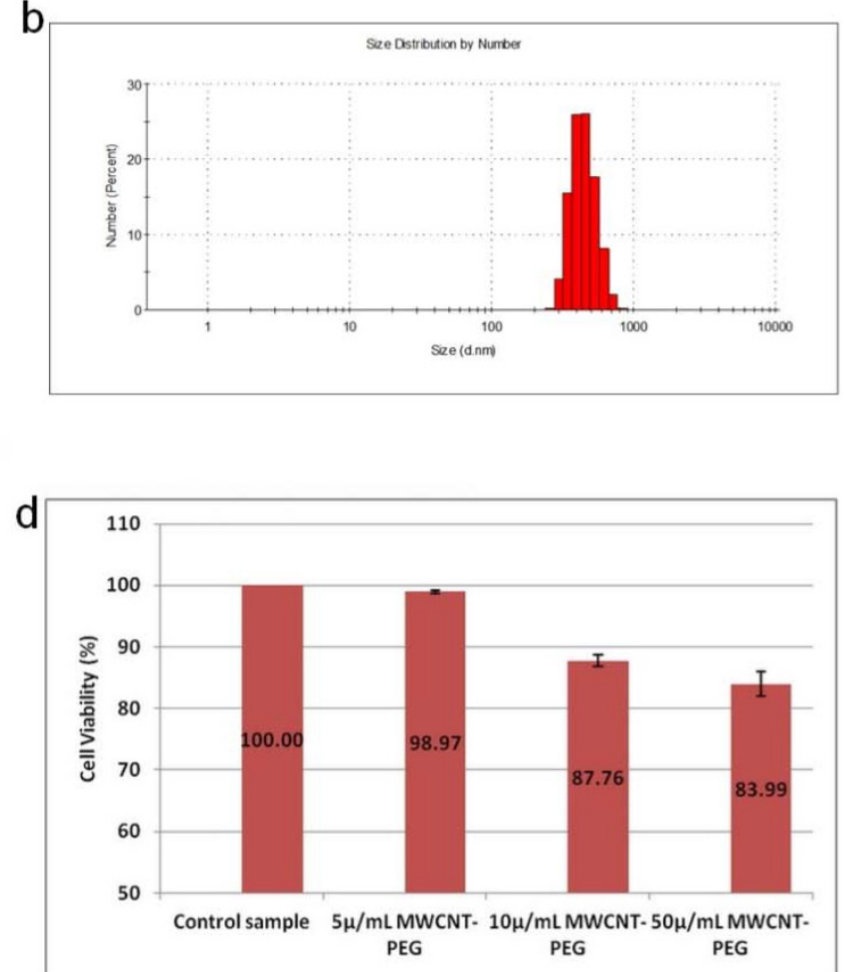

Figure 2: A: UV-Vis spectra of MWCNTs-PEG aqueous solution versus PEG solution B: DLS analysis of PEG-MWCNTs complexes. C: AFM image of a PEG functionalized MWCNT D: MTT-based determination of cell growth/viability. Control samples were exposed to normal cell culture conditions. Test groups were exposed to 5 , 10 or $50 \mu g / \mathrm{mL}$ MWCNT-PEG (I hr, $\left.37^{\circ} \mathrm{C}\right)$, followed by Laser irradiation ( $\left.3 \mathrm{~min}, 808 \mathrm{~nm}, 2 \mathrm{~W} / \mathrm{cm} 2\right)$. Abbreviations: UV-VIS-UltraViolet-Visible Spectroscopy, MWCNTs-multi-walled carbon nanotubes, AFM-atomic force microscopy, DLS- dynamic light scattering, MTTMethylthiazol Tetrazolium Assay.

\section{MWCNTs-PEG internalization inside PANC-I cells}

The ability of a MWCNTs-PEG bioconjugate to internalize inside a PANC-1 cell was evaluated by Darkfield Light Microscopy imaging. The results presented in Fig 3 show that at low concentrations and short exposure time, MWCNTs-PEG accumulates inside PANC-1 cells (white arrows). Thus, we provided imaging evidence that $\mathrm{PEG}$ may act as a carrier for MWCNTs, and because we were unable to identify any fluorescence in the epithelial cells in similar conditions (Fig.3a) we reasoned that MWCNTs-PEG bioconjugates exhibit dose dependant affinity for pancreatic cancer cells.

\section{Cell Growth Determination Kit}

Using MTT assay, following nanomediated photothermal therapy as described above a significant decrease in cell viability, as detected by their ability to form formazan was obtained for samples treated with 10 and $50 \mu \mathrm{g} / \mathrm{mL}$ as compared to control group. $(\mathrm{p}<0.05$, Fig $2 \mathrm{~d})$.

\section{Annexin V-Cy3 Apoptosis Detection Kit}

To assess the apoptotic effect of MWCNTs-PEG following Laser treatment, we examined the translocation of phosphatidyl serine (PS) groups from the inner to the exterior surface of the membrane as marker of early apoptosis state induction (19). Using the high affinity of annexin to PS groups we were able to detect the apoptosis rate in all samples. Our results (Fig 4) show a higher pro-apoptotic effect in treated samples as compared to control. Increasing effects were obtained for treatment with the increase of MWCNTs-PEG solutions 'concentration (5, 10, 50 $\mu \mathrm{g} / \mathrm{mL}$ ). Highest red staining intensity and presence (coming from $\mathrm{Cy} 3$ binded to annexin $\mathrm{V}$ ) has been observed in samples treated with $50 \mu \mathrm{g} / \mathrm{mL}$ MWCNTs-PEG followed by laser irradiation (Fig. 4). Image $J$ quantification of all fluorescent images following laser mediated treatment with MWCNTs-PEG (with concentrations above $10 \mu \mathrm{g} / \mathrm{mL}$ ) revealed a significantly increased red fluorescence suggesting strong induction of apoptosis (Chi square, $\mathrm{p}<0.05$ )

\section{Mitochondrial Membrane Potential Detection Kit}

Mitochondrial membrane potential represents an important indicator of the energetic state of the mitochondria and of the overall cellular health status (20). To test the possible involvement of mitochondrial opening of the transition pore, with cytochrome $\mathrm{C}$ release and the triggering of other downstream events in the apoptotic cascade following treatment, we 
performed analysis of mitochondrial potential using a specific assay for fluorescence microscopy. As seen in figure 5, mitochondrial potential has been distinctly decreased in test samples in a dose-dependent manner as compared to controls. Increasing depolarization has been detected with the increase of MWCNTs-PEG concentration following photothermal treatment. Image J analysis of all green fluorescent images following treatment revealed a significantly increased green fluorescence suggesting alteration of permeability transition (PT) (Chi square, $\mathrm{p}<0.05$ in all treated samples compared to controls) regardless of MWCNTs-PEG concentration.

\section{Total ROS Detection Kit}

Various stressful stimuli, such as metabolic stress and exposure to anticancer drugs radiations, or nanomaterials may induce mitochondrial dysfunction with oxidative stress (21). This process can be monitored in real time using a positively charged cell-penetrant fluorochrome that is preferentially internalized in actively respiring mitochondria in "in vitro" cultured cells. Motivated by the data sustaining
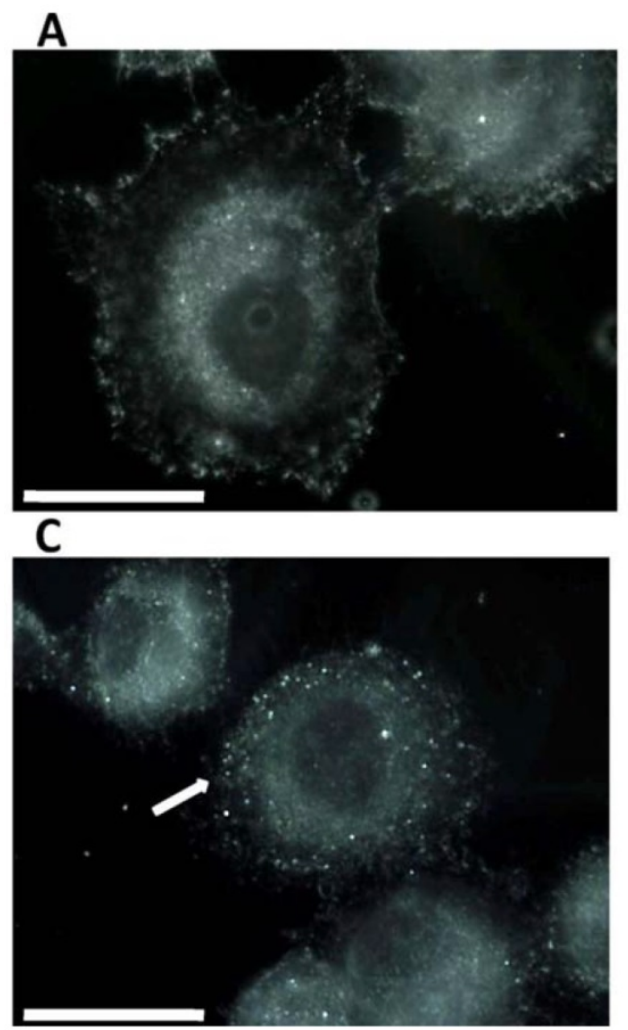

Figure 3: Dark field Light Microscopy Images of MWCNTs-PEG uptake into PANCI cell. A: Control sample (no MWCNTs-PEG exposure); B: Exposure to 5 $\mu$ g/mL MWCNTs-PEG (I hr, $\left.37^{\circ} \mathrm{C}\right)$; C: Exposure to $10 \mu \mathrm{g} / \mathrm{mL}$ MWCNTs-PEG (I hr, $\left.37^{\circ} \mathrm{C}\right)$; D: Exposure to $\left.50 \mu \mathrm{g} / \mathrm{mL} \mathrm{MWCNTs-PEG} \mathrm{(I} \mathrm{hr,} 37^{\circ} \mathrm{C}\right)$; scale bar: $50 \mu \mathrm{m}$.
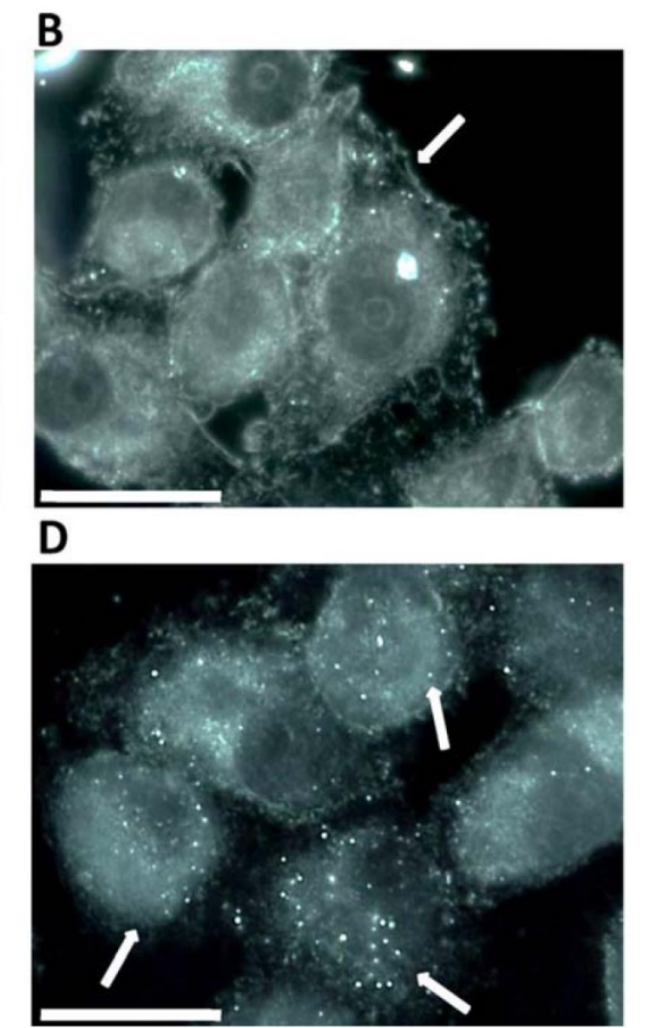

the key role of permeability transition dysfunction in cancer cell mitochondria following photothermal treatment, we next determined reactive oxygen and nitrogen species (ROS/RNS) production in PC cells. To directly monitor real time reactive oxygen and/or nitrogen species (ROS/RNS) production in PC cells following treatment, ROS staining kit for flow cytometry was used. Changes in the mitochondrial membrane potential- induce specific staining patterns which are reliable indicators of mitochondrial dysfunction (22). Therefore this assay has been increasingly used in the field of nanoparticles toxicity (23).

As seen in figure 6, flowcytometric analyzes revealed increasing FL-1 (green channel) positive cells with the increasing nanoparticle concentration. Statistics of dot-plot for forward scatter and FL-1 displayed $0.2 \%$ ROS-positive cells in control sample, $3.21 \%$ ROS-positive cells in $5 \mu \mathrm{g} / \mathrm{mL}$ MWCNTs-PEG group, $36.2 \%$ in $10 \mu \mathrm{g} / \mathrm{mL}$ MWCNTs-PEG group and $54.7 \%$ ROS-positive cells in $50 \mu \mathrm{g} / \mathrm{mL}$ MWCNTs-PEG group.

B

D 
A

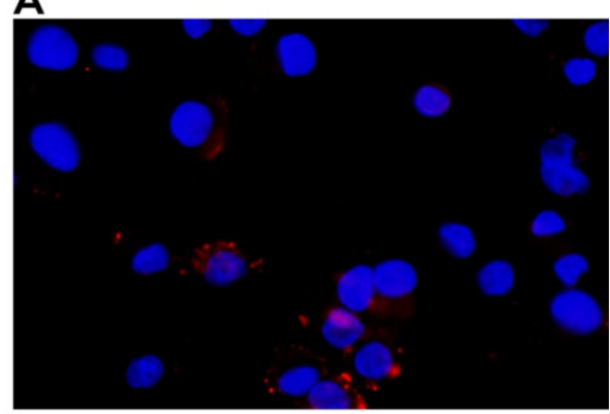

C

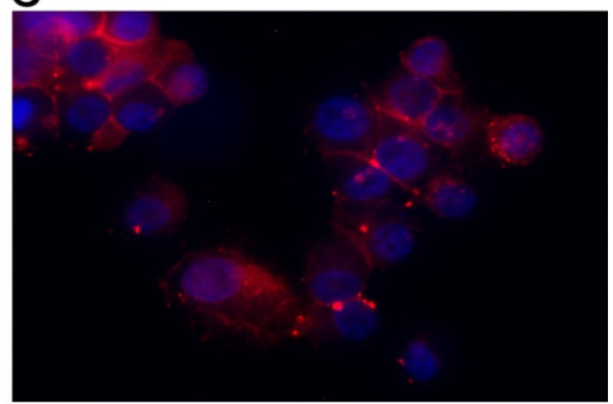

B

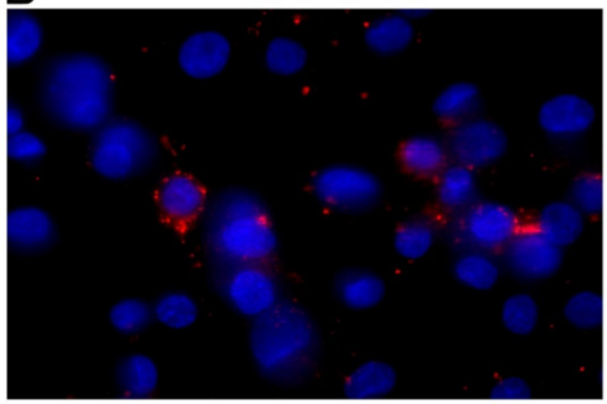

D

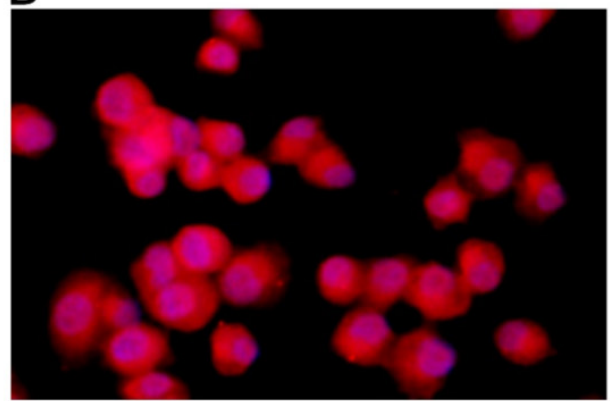

Figure 4: Detection of apoptosis using Annexin V-cy3. Control sample followed standard cell culture conditions. Test cells were treated with MWCNTs-PEG with different concentrations $(5,10$ and $50 \mu \mathrm{g} / \mathrm{mL}$, respectively) and LASER irradiated. Consequently, all samples were stained with annexin-cy3 fo 5 min (RT, dark). Nucleus staining was also performed using DAPI blue-fluorescence. A: Control sample (no MWCNTs-PEG exposure, no irradiation). Red fluorescence appears in a reduced number of cells, granular aspect coming from limited number of PS groups exposed on the outer surface of the membrane, B: Exposure to $5 \mu \mathrm{g} / \mathrm{mL} \mathrm{MWCNTs}-\mathrm{PEG}$ (I hr, $37^{\circ} \mathrm{C}$ ), followed by Laser excitation ( $3 \mathrm{~min}, 808 \mathrm{~nm}, 2 \mathrm{~W} / \mathrm{cm} 2)$. Reduced number of apoptotic cells, slight increase in the number of PS groups exposed on the surface of the membrane, C: Exposure to $10 \mu \mathrm{g} / \mathrm{mL}$ MWCNTs-PEG ( $\left.\mathrm{hr}, 37^{\circ} \mathrm{C}\right)$, followed by Laser excitation $(3 \mathrm{~min}, 808 \mathrm{~nm}, 2 \mathrm{~W} / \mathrm{cm} 2)$, Increased number of early-apoptosis entrance cells. Red fluorescence aspect is diffuse, suggesting intense PS translocation process. D: Exposure to $50 \mu \mathrm{g} / \mathrm{mL}$ MWCNTs-PEG $\left(\mathrm{I} \mathrm{hr}, 37^{\circ} \mathrm{C}\right)$, followed by Laser excitation $(3 \mathrm{~min}, 808 \mathrm{~nm}, 2 \mathrm{~W} / \mathrm{cm} 2)$. The majority of cells present intense, diffuse, red $\mathrm{Cy} 3$ fluorescence covering the entire outer surface of the membrane suggesting a highly intense pro-apoptotic effect.

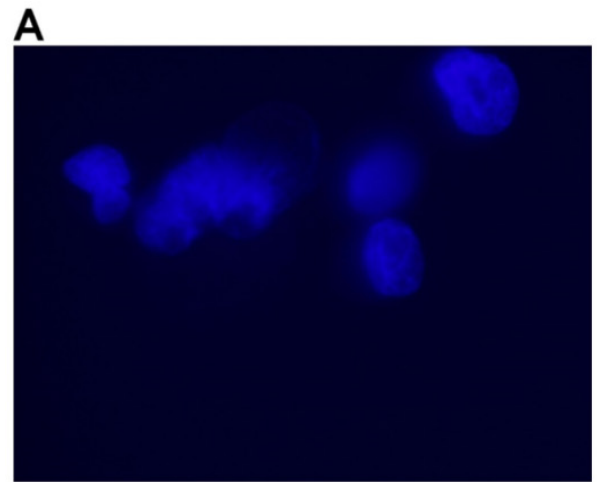

C

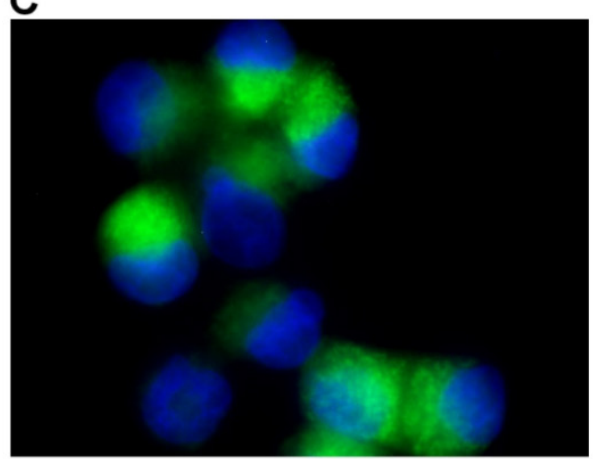

B

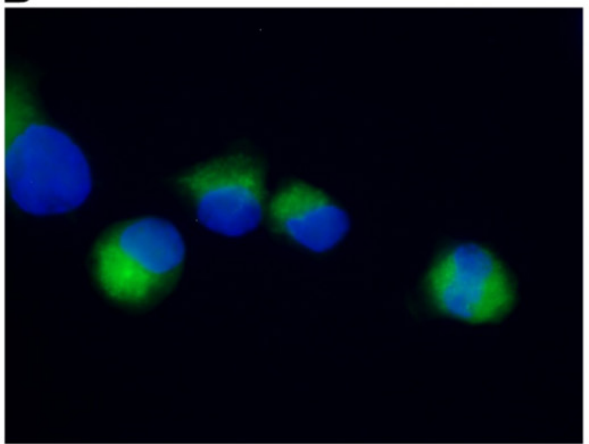

D

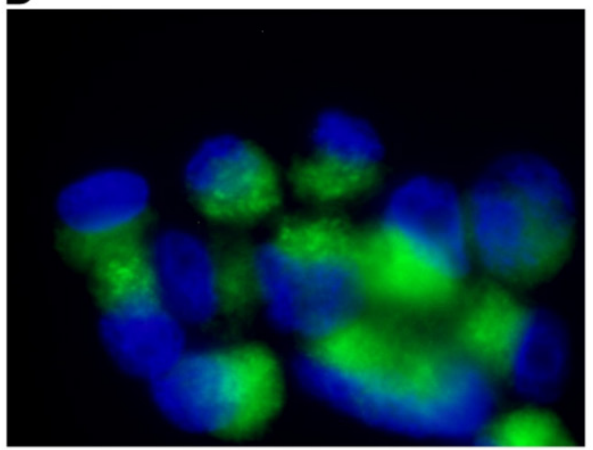

Figure 5: Detection of Mitochondrial Membrane Potential. Control sample followed standard cell culture conditions. Test cells were treated with MWCNTs-PEG with different concentrations $(5,10$ and $50 \mu \mathrm{g} / \mathrm{mL}$, respectively) and LASER irradiated. Consequently, all samples were stained with Mitochondrial Dual Detection Reagent (I5 min, RT, dark) with green fluorescence staining the cells showing mitochondrial membrane depolarizarion. Nucleus staining was also performed using DAPI blue-fluorescence. A: Control sample (no MWCNTs-PEG exposure, no irradiation) No green fluorescence is visible, suggesting membrane potential electrical equillibrium, B: Exposure to $5 \mu g / m L$

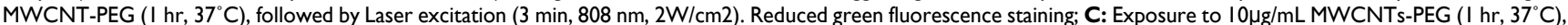
followed by Laser excitation ( $3 \mathrm{~min}, 808 \mathrm{~nm}, 2 \mathrm{~W} / \mathrm{cm} 2$ ), intense, inhomogenuous, granular aspect of green fluorescence staining; D: Exposure to $50 \mu \mathrm{g} / \mathrm{mL}$ MWCNTs-PEG (I hr, $\left.37^{\circ} \mathrm{C}\right)$, followed by Laser excitation $(3 \mathrm{~min}, 808 \mathrm{~nm}, 2 \mathrm{~W} / \mathrm{cm} 2)$. The majority of cells present green fluorescent staining as a result of mitochondrial potential drop. Aspect is intense and widely spread, with fluorescence overlap between adjacent cells. 

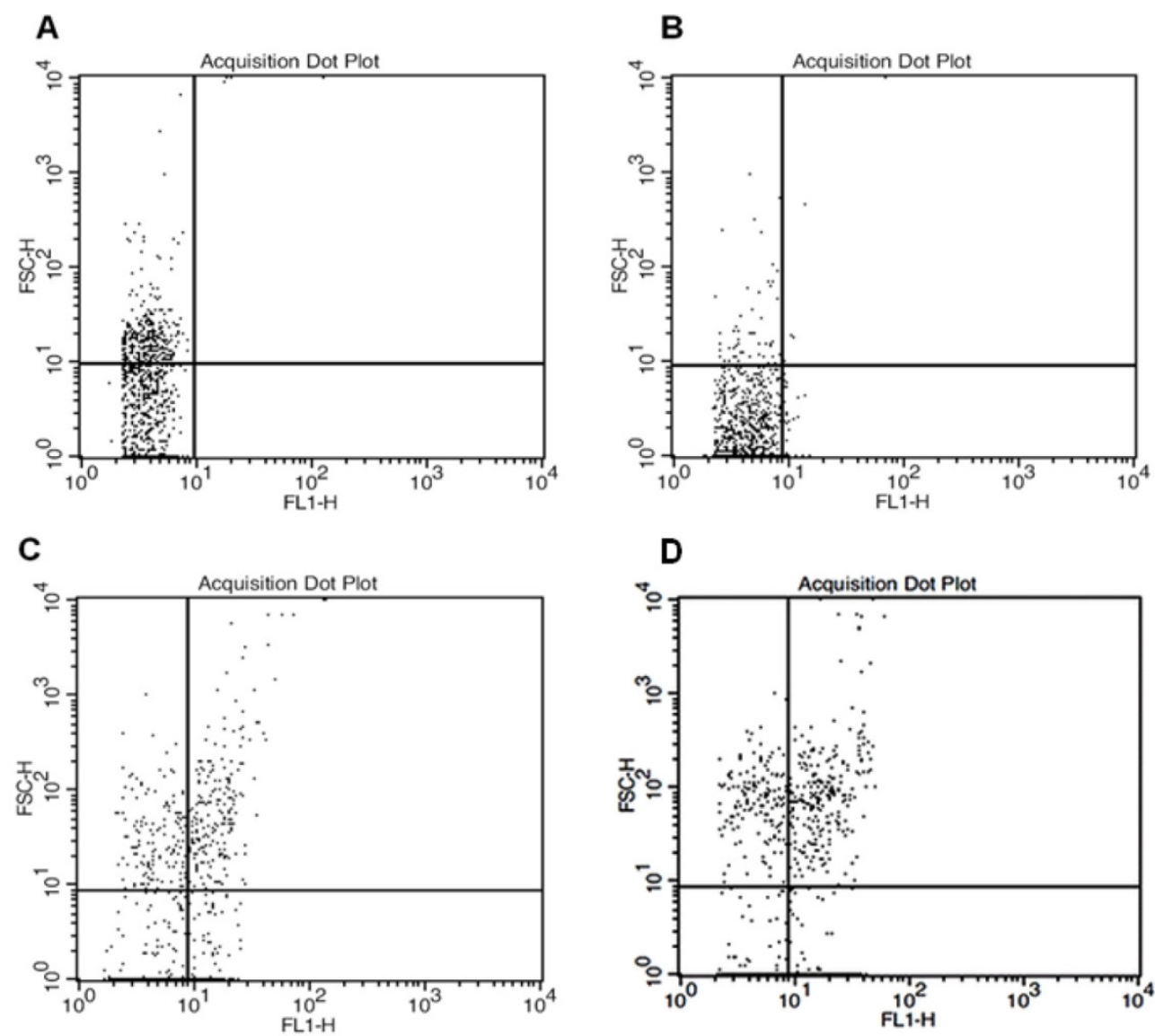

Figure 6: Flowcytometric analysis of total ROS accumulation. Forward scatter and FLI - height parameters. A: Control sample (standard cell culture conditions) B: Exposure to $5 \mu \mathrm{g} / \mathrm{mL}$ MWCNTs-PEG (I hr, $\left.37^{\circ} \mathrm{C}\right)$, followed by Laser excitation $(3 \mathrm{~min}, 808 \mathrm{~nm}, 2 \mathrm{~W} / \mathrm{cm} 2)$. C: Exposure to $10 \mu \mathrm{g} / \mathrm{mL}$ MWCNTs-PEG (I hr, $\left.37^{\circ} \mathrm{C}\right)$, followed by Laser excitation (3min, $808 \mathrm{~nm}, 2 \mathrm{~W} / \mathrm{cm} 2$ D: Exposure to $50 \mu \mathrm{g} / \mathrm{mL}$ MWCNTs-PEG $\left(1 \mathrm{hr}, 37^{\circ} \mathrm{C}\right)$, followed by Laser excitation $(3 \mathrm{~min}, 808 \mathrm{~nm}, 2 \mathrm{~W} / \mathrm{cm} 2)$.

\section{Discussions}

There is abundant literature data regarding the use of hyperthermia resulting from laser-induced carbon based materials photo-excitation to generate necrosis of malignant cells (24-27). Extensive research was also carried to elucidate the intracellular fate of internalized carbon nanotubes (28). Although the primary cell death mechanism demonstrated and aimed is thermal necrosis (29), there is less knowledge on the initial molecular mechanisms that cause death using these laser-activated nanostructures.

Nanomediated photothermal therapy represents a method of cellular necrosis at a molecular level, in which photon energy from LASER activated nanoparticles is converted to heat in cancer cells and tissue (here, temperature is elevated to $40-43^{\circ} \mathrm{C}$ and above) (30). The use of such thermally active particles in near-infrared destruction of cancer cells is highly attractive due to their facile synthesis, affordable bioconjugation, and tunable optical absorbtion $(31,32)$. In the present study, infrared absorbtion properties of carbon nanotubes were used to produce localized lysis to malign cells at sublethal levels by changes in cellular functions.
At the current time, mitochondria has become an attractive drug target in the selective treatment of malignant tumors. Different possible cancer treatment options rely on activation of mitochondrial membrane permeabilization leading to mitochondrial programmed cell death by various pharmacological agents (33). Mitochondria play a central part in cell death in response to anticancer agents. Most of these agents target mitochondria via caspases or other regulator elements of the apoptotic machinery. Nevertheless, some anticancer agents, already in clinical use (paclitaxel, vinblastine, lonidamine, etoposide, arsenic trioxide) or in pre-clinical development (betulinic acid, MT21), directly target and permeabilize mitochondria membrane (34).

By analysing mitochondrial potential and caspase activity in PC laser irradiated cells, we show here that mitochondrial membrane permeabilization with consequent apoptosis represent the primary mode of death induction following administration of MWCNTs-PEG combined with Laser irradiation by activating early apoptosis pathways. Our results are of decisive importance especially in regard to the development of novel nano-biosystems capable to target 
mitochondria and to synergically act both as cytotoxic drug as well as thermally active agents. In addition our results could lead to new research in order to overcome one of the most common problem met in oncology, that of intrinsic resistance to chemotherapeutics. Our rationale for choosing PEG in this study was its ability to act like a ligand for biofunctionalization of various nanomaterials with active drugs, peptides and hormones (35). The tested biocompound, MWCNTs-PEG, represent a precursor for many of the bio-nanosystems used today for various experimental researches in a large array of incurable diseases (36-39).

Several in vitro experiments assert that oxidative stress from CNTs is a major result of toxicity. However, most of the studies suggesting that functionalized CNTs are nontoxic in vivo outnumbers those proposing otherwise. This fact suggests that varying functionalization can modify toxicity (8).

From a clinical point of view, the CNTs mediated nanophotothermolysis of tumor lessions implies the ultrasound identification of the malign tissue and its vascular supply, intra-arterial administration of the functionalized CNTs, followed by laser irradiation. Using minimally invasive surgery, such as laparoscopic approach it is possible to identify the main artery supply of the tumor and to intraarterially administer carbon nanotubes solution followed by laser irradiation with major benefits for the patient.(9)

\section{Conclusions}

We have shown here that hyperthermia following MWCNTs-PEG laser mediated treatment activates the flux of free radicals within the cell and the oxidative state mediate cellular damage in PC cells via apoptotic pathway. Thus, using MWCNTs-PEG and LASER we were able to alter the redox equilibrium and induce an intense oxidative state in the pancreatic malign cell.

Further research is required to fully understand the whole spectrum of molecular and cellular interactions in order to develop novel hybrid drugs that contain both thermally active nanomaterials, as well as apoptotic substances for the treatment of cancer. Our results could have a very significant impact on the design of nanotherapeutics vectors that modulates apoptosis. Nevertheless, further investigations are needed for the careful assessment of unexpected toxicities and biological interactions of carbon nanotubes bioconjugates inside the living organism.

\section{Competing Interests}

None to declare.

\section{Acknowledgements}

This work was funded by the Romanian Ministry of Education and Research (PN-II-RUTE-2011-3-0251; PN-II-PT-PCCA-2011-3.1-1551; PN-IIPT-PCCA-2011-3.1-1586; PN-II-ID-PCE-2012-4-0243; PN-II-ID-PCE-2012-4-0241 ).

\section{Authors' contributions}

T.M and I.C. performed immuncytochemistry and cell proliferation studies, writing various sections of the manuscript, C.M. performed biofunctionalization of MWCNTs with PEG and their characterization, I.I. and F.T performed flowcytometric analysis as well as fluorescence microscopy analysis, C.I. designed the study; L.M. supervised the research and wrote the manuscript.

\section{References}

1. Jemal A, Bray F, Center MM, Ferlay J, Ward E, Forman D. Global cancer statistics. CA Cancer J Clin 2011; 61(2): 69-90.

2. Borja-Cacho D, Jensen EH, Saluja AK, Buchsbaum DJ, Vickers SM. Molecular targeted therapies for pancreatic cancer. Am J Surg 2008; 196(3): 430-441.

3. Mocan L, Ilie I, Tabaran FA, Dana B, Zaharie F, Zdrehus C, Puia C, et al. Surface plasmon resonance-induced photoactivation of gold nanoparticles as mitochondria-targeted therapeutic agents for pancreatic cancer. Expert opinion on therapeutic targets 2013; (0): 1-11.

4. Mulvey JJ, Villa $\mathrm{CH}$, McDevitt MR, Escorcia FE, Casey E, Scheinberg DA. Self-assembly of carbon nanotubes and antibodies on tumours for targeted amplified delivery. Nature nanotechnology 2013; 8(10): 763-771.

5. Iverson NM, Barone PW, Shandell M, Trudel LJ, Sen S, Sen F, Ivanov V, et al. In vivo biosensing via tissue-localizable near-infrared-fluorescent single-walled carbon nanotubes. Nature nanotechnology 2013; 8(11): 873-880.

6. Iancu C, Mocan L, Bele C, Orza AI, Tabaran FA, Catoi C, Stiufiuc R, et al. Enhanced laser thermal ablation for the in vitro treatment of liver cancer by specific delivery of multiwalled carbon nanotubes functionalized with human serum albumin. International Journal of Nanomedicine 2011; 6: 129.

7. Huang N, Wang $\mathrm{H}$, Zhao J, Lui $\mathrm{H}$, Korbelik M, Zeng H. Single-wall carbon nanotubes assisted photothermal cancer therapy: Animal study with a murine model of squamous cell carcinoma. Lasers Surg Med 2010; 42(9): 638-648.

8. Iancu C, Mocan L. Advances in cancer therapy through the use of carbon nanotube-mediated targeted hyperthermia. International Journal of Nanomedicine 2011; 6: 1675 .

9. Antaris AL, Robinson JT, Yaghi OK, Hong G, Diao S, Luong R, Dai H. Ultra-low doses of chirality sorted $(6,5)$ carbon nanotubes for simultaneous tumor imaging and photothermal therapy. ACS nano 2013; 7(4): 3644-3652.

10. Mittal S, Sharma V, Vallabani NV, Kulshrestha S, Dhawan A, Pandey AK. Toxicity evaluation of carbon nanotubes in normal human bronchial epithelial cells. J Biomed Nanotechnol 2011; 7(1): 108-109.

11. Toyokuni S. Genotoxicity and carcinogenicity risk of carbon nanotubes. Adv Drug Deliv Rev 2013; 65(15): 2098-2110.

12. Patlolla A, McGinnis B, Tchounwou P. Biochemical and histopathological evaluation of functionalized single-walled carbon nanotubes in swiss-webster mice. J Appl Toxicol 2011; 31(1): 75-83.

13. Tu X, Ma Y, Cao Y, Huang J, Zhang M, Zhang Z. PEGylated carbon nanoparticles for efficient in vitro photothermal cancer therapy. Journal of Materials Chemistry B 2014; 2:2184-2192.

14. Song M, Zeng L, Yuan S, Yin J, Wang H, Jiang G. Study of cytotoxic effects of single-walled carbon nanotubes functionalized with different chemical groups on human MCF7 cells. Chemosphere 2013; 92(5): 576-582.

15. Ilie I, Ilie R, Mocan T, Tabaran F, Iancu C, Mocan L. Nicotinamide-functionalized multiwalled carbon nanotubes increase insulin production in pancreatic beta cells via MIF pathway. International Journal of Nanomedicine 2013; 8: 3345-3353.

16. Botelho EC, Edwards ER, Bittmann B, Burkhart T. Dispersing carbon nanotubes in phenolic resin using an aqueous solution. Journal of the Brazilian Chemical Society 2011; 22(11): 2040-2047.

17. Smith B, Wepasnick K, Schrote K, Bertele A, Ball WP, O'Melia C, Fairbrother DH. Colloidal properties of aqueous suspensions of acid-treated, multi-walled carbon nanotubes. Environ Sci Technol 2008; 43(3): 819-825.

18. Moon YK, Lee J, Lee JK, Kim TK, Kim SH. Synthesis of length-controlled aerosol carbon nanotubes and their dispersion stability in aqueous solution. Langmuir 2009; 25(3): 1739-1743.

19. Blankenberg FG, Norfray JF. Multimodality molecular imaging of apoptosis in oncology. Am J Roentgenol 2011; 197(2): 308-317. 
20. Perry SW, Norman JP, Barbieri J, Brown EB, Gelbard HA. Mitochondrial membrane potential probes and the proton gradient: A practical usage guide. BioTechniques 2011; 50(2): 98-115.

21. Mocan T, Clichici S, Biris A, Simon S, Catoi C, Tabaran F, Filip A, et al. Dynamic effects over plasma redox balance following subcutaneous injection of single walled carbon nanotubes functionalized with single strand DNA. Dig J Nanomat Bios 2011; 6(3):1207-1214.

22. Boren J, Brindle K. Apoptosis-induced mitochondrial dysfunction causes cytoplasmic lipid droplet formation. Cell Death \& Differentiation 2012; 19(9): 1561-1570.

23. Soenen SJ, Rivera-Gil P, Montenegro J, Parak WJ, De Smedt SC, Braeckmans K. Cellular toxicity of inorganic nanoparticles: Common aspects and guidelines for improved nanotoxicity evaluation. Nano Today 2011; 6(5): 446-465.

24. Shen S, Ren J, Zhu X, Pang Z, Lu X, Deng C, Zhang R, et al. Monodisperse magnetites anchored onto carbon nanotubes: A platform for cell imaging, magnetic manipulation and enhanced photothermal treatment of tumors. Journal of Materials Chemistry B 2013; 1(14): 1939-1946.

25. Yang K, Zhang S, Zhang G, Sun X, Lee S, Liu Z. Graphene in mice: Ultrahigh in vivo tumor uptake and efficient photothermal therapy. Nano letters 2010; 10(9): 3318-3323.

26. Liu Z, Robinson JT, Tabakman SM, Yang K, Dai H. Carbon materials for drug delivery \& cancer therapy. Materials today 2011; 14(7): 316-323.

27. Ma X, Tao H, Yang K, Feng L, Cheng L, Shi X, Li Y, et al. A functionalized graphene oxide-iron oxide nanocomposite for magnetically targeted drug delivery, photothermal therapy, and magnetic resonance imaging. Nano Research 2012; 5(3): 199-212.

28. Zhou F, Xing D, Wu B, Wu S, Ou Z, Chen WR. New insights of transmembranal mechanism and subcellular localization of noncovalently modified single-walled carbon nanotubes. Nano letters 2010; 10(5): 1677-1681.

29. Chou H, Wang T, Lee C, Tai N, Chang H. Photothermal effects of multi-walled carbon nanotubes on the viability of BT-474 cancer cells. Materials Science and Engineering: C 2013; 33(2): 989-995.

30. Mocan L, Tabaran F, Mocan T, Bele C, Orza A, Lucan C, Stiufiuc R, et al. Selective ex-vivo photothermal ablation of human pancreatic cancer with albumin functionalized multiwalled carbon nanotubes. International Journal of Nanomedicine 2011; 6(1): 915-928.

31. Vashist SK, Zheng D, Pastorin G, Al-Rubeaan K, Luong JH, Sheu F. Delivery of drugs and biomolecules using carbon nanotubes. Carbon 2011; 49(13): 4077-4097.

32. Andersen AJ, Robinson JT, Dai H, Hunter AC, Andresen TL, Moghimi SM. Single-walled carbon nanotube surface control of complement recognition and activation. ACS nano 2013; 7(2): 1108-1119.

33. Fulda S, Galluzzi L, Kroemer G. Targeting mitochondria for cancer therapy. Nature reviews Drug discovery 2010; 9(6): 447-464

34. Biswas S, Dodwadkar NS, Deshpande PP, Torchilin VP. Liposomes loaded with paclitaxel and modified with novel triphenylphosphonium-PEG-PE conjugate possess low toxicity, target mitochondria and demonstrate enhanced antitumor effects $<\mathrm{i}>$ in vitro $</ \mathrm{i}>$ and $<\mathrm{i}>$ in vivo $</ \mathrm{i}>$. J Controlled Release 2012; 159(3): 393-402.

35. Sacchetti C, Rapini N, Magrini A, Cirelli E, Bellucci S, Mattei M, Rosato N, et al. In vivo targeting of intratumor regulatory $\mathrm{T}$ cells using PEG-modified single-walled carbon nanotubes. Bioconjug Chem 2013; 24(6): 852-858.

36. Niu L, Meng L, Lu Q. Folate-Conjugated PEG on single walled carbon nanotubes for targeting delivery of doxorubicin to cancer cells. Macromolecular bioscience 2013; 13(6): 735-744.

37. Das M, Singh RP, Datir SR, Jain S. Intranuclear drug delivery and effective in vivo cancer therapy via Estradiol-PEG-appended multiwalled carbon nanotubes. Molecular pharmaceutics 2013; 10(9): 3404-3416.

38. Cheng Z, Chai R, Ma P, Dai Y, Kang X, Lian H, Hou Z, et al. Multiwalled carbon nanotubes and NaYF4: Yb3 / Er3 nanoparticle-doped bilayer hydrogel for concurrent nir-triggered drug release and up-conversion luminescence tagging. Langmuir 2013; 29(30): 9573-9580.

39. Lee P, Chiou Y, Wong J, Peng C, Shieh M. Targeting colorectal cancer cells with single-walled carbon nanotubes conjugated to anticancer agent SN-38 and EGFR antibody. Biomaterials 2013; 34(34): 8756-8765. 